\title{
Deep brain stimulator implantation in a diagnostic MRI suite: infection history over a 10 -year period
}

\author{
Alastair J. Martin, PhD, ${ }^{1}$ Paul S. Larson, MD, ${ }^{2}$ Nathan Ziman, BS, ${ }^{3}$ Nadja Levesque, RN, ${ }^{2}$ \\ Monica Volz, RN, ${ }^{3}$ Jill L. Ostrem, MD, ${ }^{3}$ and Philip A. Starr, MD, PhD² \\ Departments of ${ }^{1}$ Radiology and Biomedical Imaging, ${ }^{2}$ Neurological Surgery, and ${ }^{3}$ Neurology, University of California, San \\ Francisco, California
}

\begin{abstract}
OBJECTIVE The objective of this study was to assess the incidence of postoperative hardware infection following interventional (i)MRI-guided implantation of deep brain stimulation (DBS) electrodes in a diagnostic MRI scanner.

METHODS A diagnostic 1.5-T MRI scanner was used over a 10-year period to implant DBS electrodes for movement disorders. The MRI suite did not meet operating room standards with respect to airflow and air filtration but was prepared and used with conventional sterile procedures by an experienced surgical team. Deep brain stimulation leads were implanted while the patient was in the magnet, and patients returned 1-3 weeks later to undergo placement of the implantable pulse generator (IPG) and extender wire in a conventional operating room. Surgical site infections requiring the removal of part or all of the DBS system within 6 months of implantation were scored as postoperative hardware infections in a prospective database.

RESULTS During the 10-year study period, the authors performed 164 iMRI-guided surgical procedures in which 272 electrodes were implanted. Patients ranged in age from 7 to 78 years, and an overall infection rate of $3.6 \%$ was found. Bacterial cultures indicated Staphylococcus epidermis (3 cases), methicillin-susceptible Staphylococcus aureus (2 cases), or Propionibacterium sp. (1 case). A change in sterile practice occurred after the first 10 patients, leading to a reduction in the infection rate to $2.6 \%$ ( 4 cases in 154 procedures) over the remainder of the procedures. Of the 4 infections in this patient subset, all occurred at the IPG site.
\end{abstract}

CONCLUSIONS Interventional MRI-guided DBS implantation can be performed in a diagnostic MRI suite with an infection risk comparable to that reported for traditional surgical placement techniques provided that sterile procedures, similar to those used in a regular operating room, are practiced.

http://thejns.org/doi/abs/10.3171/2015.7.JNS15750

KEY WORDS DBS; infection; MRI; functional neurosurgery; deep brain stimulation

I NTRAOPERATIVE MRI has been used for a range of neurosurgical procedures such as tumor resection monitoring, biopsy, and laser ablation. ${ }^{6}$ Over the past 10 years, we have pioneered the use of interventional (i)MRI techniques to implant deep brain stimulation (DBS) electrodes..$^{10,11,16}$ Precise electrode placement within a selected brain region is necessary to achieve efficacy, which is traditionally done with frame-based or "frameless" neuronavigation-guided stereotaxy supported by invasive physiological testing including microelectrode recording. Physiological methods can be used to mitigate the accuracy limitations of stereotactic methods that use a skull-mounted frame or other external markers for spatial registration of brain structures to preoperatively acquired images. $^{2}$
The iMRI DBS electrode implantation technique utilizes bur hole-mounted trajectory guides oriented toward targets that are defined intraoperatively. The entire procedure is performed within the bore of an MR magnet, with the patient's head positioned at the rear magnet opening for surgical exposure and at the magnet isocenter for target identification, device alignment, and insertion monitoring. The primary benefits of the iMRI technique are high targeting accuracy, reduced operative time, and an ability to directly confirm electrode placement during the procedure. In contrast to conventional stereotactic methods, the iMRI approach is performed with the patient under general anesthesia and does not require intraoperative testing of motor symptoms. For the most common DBS indication,

ABBREVIATIONS DBS = deep brain stimulation; iMRI = interventional MRI; IPG = implantable pulse generator; MSSA = methicillin-susceptible Staphylococcus aureus. ACCOMPANYING EDITORIAL See pp 106-107. DOI: 10.3171/2015.9.JNS151699.

SUBMITTED April 2, 2015. ACCEPTED July 21, 2015.

INCLUDE WHEN CITING Published online March 25, 2016; DOI: 10.3171/2015.7.JNS15750. 
Parkinson's disease, the iMRI method obviates the need for medication withdrawal prior to surgery.

The technique has proven to be accurate and requires only a single brain penetration in a high proportion of cases. ${ }^{16}$ The technical success of the method must be balanced against its complication rate. Hardware infection is one of the more common complications following DBS surgery. ${ }^{1,5}$ Recent studies have reported hardware infection rates ranging from $2 \%$ to $10 \%$ following conventional DBS surgery. ${ }^{1,5,14,17,18}$ Utilization of the iMRI surgical approach has the potential to increase the rate of hardware infection, especially when performed in a diagnostic MRI suite that does not meet all the sterile characteristics of an operating room. We report our incidence of hardware infections from iMRI-guided DBS electrode implantations using a diagnostic MRI scanner in a radiology suite.

\section{Methods}

Interventional MRI-guided DBS electrode implantations were performed in a diagnostic MRI suite over a period spanning from April 2004 to October 2014. All procedures were performed using institutional review board-approved protocols during the 10-year study period. The data used in this study were obtained during the clinical trial registered with clinicaltrials.gov (NCT00792532).

\section{Characteristics of the MRI Suite}

The iMRI facility features a 1.5-T MR system (Achieva, Philips) with a bore measuring $60 \mathrm{~cm}$ in diameter and $175 \mathrm{~cm}$ in length. The magnet is centered in a radiofrequency-shielded room measuring $7.7 \mathrm{~m}$ in length and 5.7 $\mathrm{m}$ in width $\left(43.9 \mathrm{~m}^{2}\right)$. The room features a suspended tile ceiling, vinyl flooring, painted drywall, and a number of wood veneer cabinets along the walls. The room volume measures $108 \mathrm{~m}^{3}$ and was evaluated for its airflow characteristics. The air supply was not HEPA filtered, and the room included 2 central air supplies and 2 return air portals at opposite ends of the room. The air supply near the rear magnet opening, where the surgical procedures were performed, was modified by mounting a simple sheet of plastic over the opening to direct this unfiltered air away from the surgical field. The room had a slight positive pressure of $0.87 \mathrm{~Pa}$, and air exchanges were measured to be 12.6/hour (Technical Safety Services Inc.). Airborne nonviable particle counts established the room as International Standardization Organization (ISO) 8.7 These properties did not meet the operating room requirements of HEPA filtration and $>25$ air exchanges/hour and were slightly below the 15 air exchanges/hour recommended for procedure rooms. ${ }^{13}$ The suite did meet the standards established for diagnostic and treatment radiology suites and emergency room trauma bays.

\section{Sterile Practices}

In addition to the redirection of unfiltered airflow away from the operative field, a number of supplementary sterile practices were followed. Prior to the procedures, all unnecessary equipment was removed from the magnet room, and the room surfaces, including the magnet, cabinets, and floors, were wiped with germicidal dispos- able wipes (Sani-Cloth AF3, PDI Inc.). The room was then considered sterile, and personnel entering the suite had to wear surgical scrubs and cap. A face mask was required beginning at the time of opening sterile surgical supplies. Patients were introduced into the MRI suite while under general anesthesia and with the hair over the immediate surgical area clipped. Patients were advanced through the magnet bore on the MR table top, such that their head extended beyond the rear magnet opening. The surgical site was scrubbed with an iodinated scrub brush, then swabbed with an iodinated solution $(7.5 \%$ povidoneiodine or iodine povacrylex with isopropyl alcohol) and allowed to dry for a minimum of 3 minutes. A layer of adhesive antimicrobial film (Ioban, 3M Inc.) was affixed to the surgical area of the scalp. A custom sterile drape (MR Neuro Drape Tapered, MRI Interventions) was then established (Fig. 1) to cover the rear magnet face and create an expandable tunnel so the patient could be moved between the surgical position and the magnet isocenter. A prophylactic dose of 1-2 $\mathrm{g}$ of intravenous cefazolin (or other antiStaphylococcus antibiotic) was administered prior to skin incision and repeated again if the surgical time exceeded 4 hours. Prophylactic antibiotics were continued postoperatively in all patients according to standard practice at the time. Early in this patient cohort, antibiotics were typically continued for 24 hours after surgery. In more recent years, however, the standard practice has changed to a single dose postoperatively.

\section{Surgical Team and Procedure}

The surgical team always included an experienced DBS surgeon, who was supported by scrub and circulating nurses with extensive operating room experience and specific expertise in conventional DBS surgery. Staffing also included an MR technologist, a neurosurgical fellow, and an MR physicist. The surgical procedure has been described in detail elsewhere. ${ }^{15,16}$ Briefly, skin incision was followed by drilling unilateral or bilateral $15-\mathrm{mm}$ frontal bur holes. Bilateral or unilateral trajectory guides (NexFrame, Medtronic, or ClearPoint SmartFrame, MRI Interventions) were fixed over the resulting opening by using temporary bone screws, and the patient was moved to the magnet isocenter. Magnetic resonance imaging was then performed to identify the desired deep brain target, orient the trajectory guides, and monitor the insertion process. Medtronic DBS electrodes (model $3389-28$ or 3389-40) were used for all studies in all patients. After the DBS electrode(s) were successfully positioned, the patient was returned to the rear magnet opening (Fig. 1), the DBS leads were anchored to the skull with the Stimloc device (Medtronic), and the trajectory guides were removed. Scalp closure was performed using conventional sutures and staples. The implantable pulse generator (IPG) and extender wire were placed in a separate surgical procedure performed in a regular operating room, typically $1-3$ weeks following DBS electrode implantation.

\section{Patients and Follow-Up}

Patient diagnoses included idiopathic Parkinson's disease, primary or secondary dystonia, Tourette's syndrome, and nonparkinsonian tremor disorders. All diagnoses were 


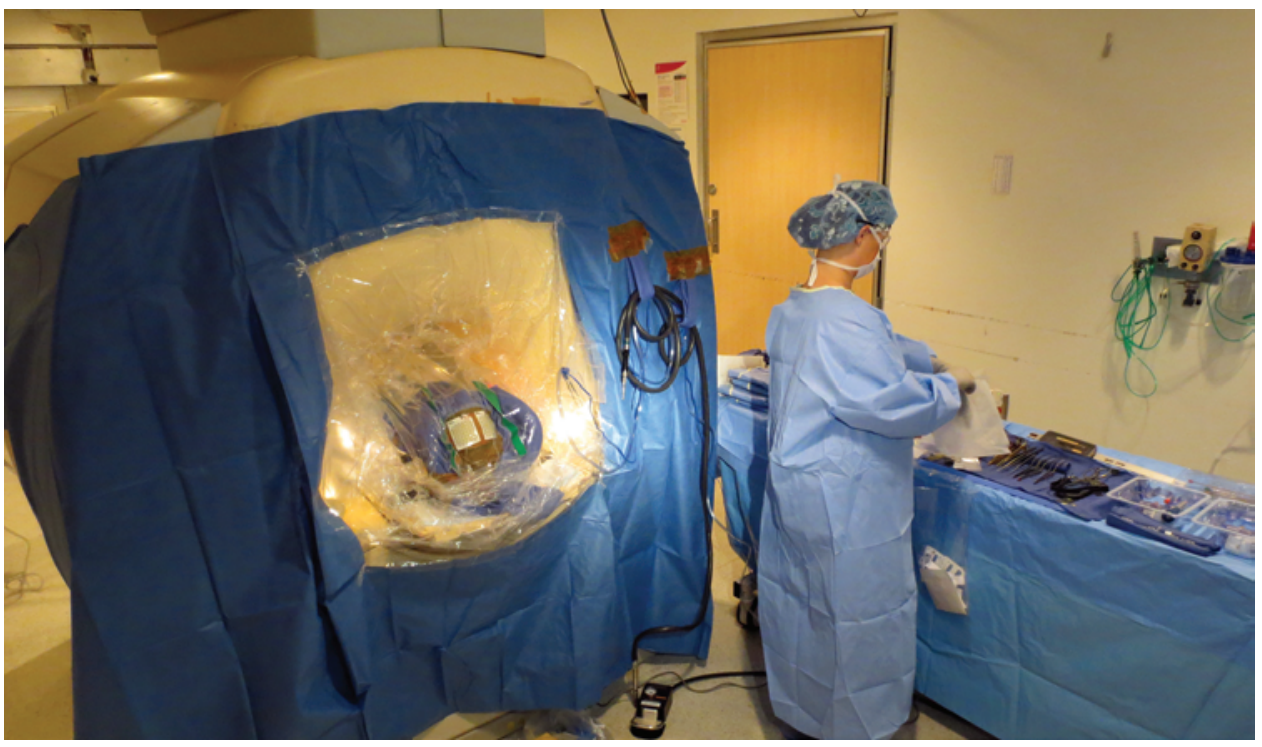

FIG. 1. The iMRI suite with sterile drapes at the rear magnet opening. The sterile drape utilizes an elastic cord system that holds the drape open when the patient moves between the surgical position (shown here) and the magnet isocenter. Figure is available in color online only.

made by a movement disorder neurologist and were deemed appropriate for DBS therapy. Inclusion and exclusion criteria were comparable to DBS candidates being screened for conventional DBS implantation. For adults, selection of the iMRI-guided DBS procedure over standard physiologically guided DBS implantation in a regular operating room was based on patient preference. For pediatric patients (age $<18$ years), the recommended method was the iMRI method for all patients after July 2010. This study was limited to patients undergoing initial DBS placement; no reoperative DBS patients were included. After surgery, patients returned for routine follow-up and IPG programming. Hardware infection occurring within 6 months of implantation was attributed to the surgical procedure. Intraoperative microbial culture was performed in all patients who underwent hardware removal for suspected infection. Minor superficial skin erythema or other findings suspicious for infection that resolved with local care or empirical oral antibiotics alone were not systematically tracked.

\section{Statistical Analysis}

Probability assessments were used to probe confidence values for the upper bound of our infection rates based on the number of infections seen $(r)$ and the number of procedures performed $(n)$. The probability of seeing " $r$ " or fewer infections in " $n$ " procedures was calculated as

$$
\sum_{x=0}^{r} n C x \times P^{x} \times(1-P)^{n-x}
$$

where ${ }_{n} C_{x}$ is the number of different unordered combinations of " $x$ " events in " $n$ " opportunities and $P$ is the true probability of infection. ${ }_{n} C_{x}$ can be calculated as

$$
{ }_{n} C_{x}=n ! /(x !(n-x) !) s
$$

In this analysis, a range of "true" infection rates were tested, and the probability of seeing the number of infections reported in our study was assessed for each putative true rate. The upper boundary of the underlying true rate was the rate at which the probability of observing the actual number of infections dropped below $5 \%$.

\section{Results}

Two attending surgeons performed all 164 iMRI-guided surgical procedures over the 10-year study period. A total of 272 electrodes were implanted in 108 bilateral and 56 unilateral procedures. Patients ranged in age from 7 to 78 years (mean \pm SD, $57 \pm 15$ years) and included 141 patients with Parkinson's disease, 19 with dystonia, 2 with Tourette's syndrome, and 2 with nonparkinsonian tremor disorders. Ten patients were younger than 18 years of age. Forty-nine implantations were performed with the NexFrame system (33 bilateral, 16 unilateral), and 115 were performed with the ClearPoint platform (75 bilateral, 40 unilateral). The transition between these systems occurred in mid-2010. Six (3.6\%) hardware-related infections occurred during the 10-year enrollment period (Table 1). All infections occurred in patients undergoing bilateral electrode implantations.

There was an important change in our clinical practice after our first 10 surgical procedures. In these early cases we did not have an MR-compatible drill; thus, the bur hole had to be prepared outside the magnet room in a separate sterile field. The patient was then transferred into the magnet suite, a new sterile field was created, and the iMRIguided procedure was performed. This technique was associated with 2 early infections that both presented within 2 weeks of surgery. Bacterial cultures indicated Staphylococcus epidermis in 1 case and Propionibacterium in the other. Both featured redness over the frontal incision site (Fig. 2) and required the removal of all implanted hard- 
TABLE 1. Summary of hardware infections over a 10-year period of iMRI-guided DBS electrode implantations

\begin{tabular}{|c|c|c|c|c|c|c|}
\hline Op Date & $\begin{array}{l}\text { Patient } \\
\text { Age (yrs) }\end{array}$ & $\begin{array}{l}\text { Time Btwn Infection } \\
\text { Symptoms \& Op (days) }\end{array}$ & Infection Description & Culture Findings & Outcome & $\begin{array}{l}\text { Hypothesized } \\
\text { Origin }\end{array}$ \\
\hline $12 / 2004$ & 45 & 9 & Redness of scalp incision site & Staphylococcus epidermis & Explanted full system & Scalp incision \\
\hline $3 / 2005$ & 76 & 13 & Redness of scalp incision site & Propionibacterium sp. (rare) & Explanted full system & Scalp incision \\
\hline $9 / 2007$ & 66 & 105 & Redness over It chest incision & Staphylococcus epidermis & Explanted full system & IPG \\
\hline $8 / 2008$ & 67 & 16 & Redness \& swelling over IPG & Staphylococcus epidermis & $\begin{array}{l}\text { IPG \& extender wires } \\
\text { replaced }\end{array}$ & IPG \\
\hline $12 / 2010$ & 61 & 92 & Redness over IPG site & MSSA & Explanted full system & IPG \\
\hline $9 / 2014$ & 52 & 16 & Pain \& swelling over IPG & MSSA & Explanted full system & IPG \\
\hline
\end{tabular}

MSSA = methicillin-susceptible Staphylococcus aureus.

ware. The patient in 1 of these cases developed cerebritis and had a long intensive care unit stay before eventually making a full recovery. These experiences led to a halt in the iMRI-guided implantation approach until an MRcompatible drill could be obtained.

The single sterile field approach (Fig. 3) was used for all subsequent procedures, which reduced the infection rate for our more current surgical practice to 4 (2.6\%) of 154. All infection symptoms in this subset of patients originated at the IPG site and not the frontal incision. This led us to hypothesize that the source of these infections was not the DBS electrode implantation, but rather the IPG implantation procedure that was performed 1-3 weeks later. In 1 of these infections only the IPG and lead extensions were explanted and subsequently replaced, with a successful outcome. In the 2 cases in which there was a severalmonth delay between surgery and infection symptoms, the patients were initially treated with antibiotics, but this ultimately proved ineffective, and explantation of all hardware was required. The 4 infections occurring after we altered our clinical practice produced bacterial cultures indicating either S. epidermis (2 cases) or methicillinsusceptible Staphylococcus aureus (MSSA; 2 cases). We have recently begun to obtain nasal swabs from patients prior to surgery to screen for Staphylococcus spp., and our

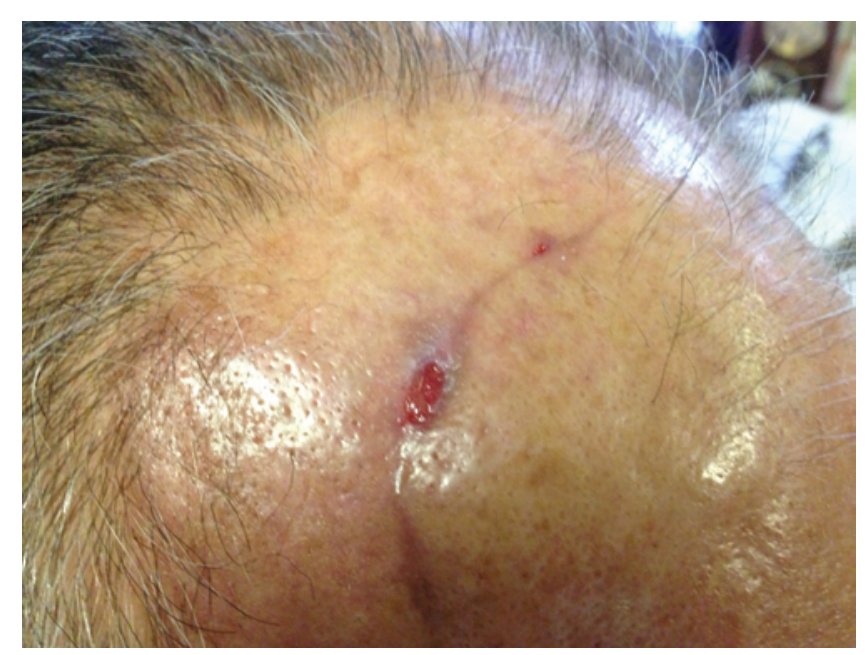

FIG. 2. Nonhealing frontal wound with drainage from the bur hole cover. Figure is available in color online only. most recent infection (9/2014) revealed the presence of MSSA 4 months prior to surgery. The patient in this case, however, had not received special antibiotic treatments leading up to surgery.

\section{Statistical Interpretation}

In this study we found 4 infections in the 154 procedures $(2.6 \%)$ performed using our current surgical practice. Probability analysis was used to evaluate upper bounds to our infection rate based on these initial data. This analysis indicated that there is a $>95 \%$ chance that our true infection rate is $<6 \%$. This upper boundary is lower if we postulate that all infections in the cases performed using our current surgical practice arose from the pulse generator implantation, which was performed in a regular operating room and was not part of the iMRIguided lead implantation. The observation of 0 infections in the iMRI-guided procedure is then associated with a $>$ $95 \%$ chance of a true infection rate $<2 \%$.

\section{Discussion}

In this study we analyzed the incidence of infection after implanting DBS electrodes using iMRI in a diagnostic MRI suite. The suite differed from a standard operating

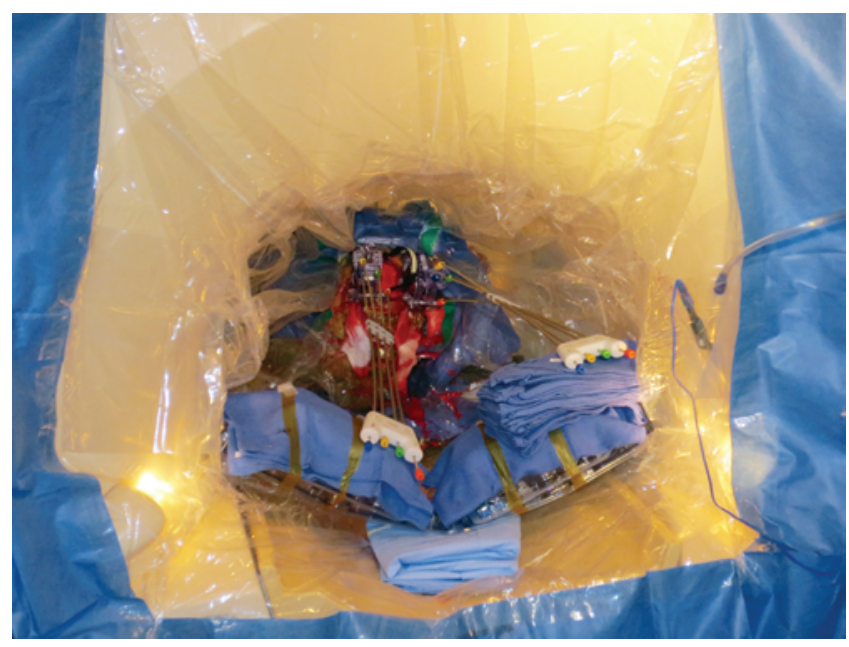

FIG. 3. The patient is at the magnetic isocenter, with bilateral trajectory guides fixed to bur holes. The sterile drape conforms to the magnet bore to maintain open sterile access. Figure is available in color online only. 
room in that it did not meet operating room requirements with respect to air filtration and airflow. The study covered a period of 10 years during which 164 surgical procedures were performed and 272 DBS electrodes were implanted. Hardware infection within 6 months of surgery occurred in 6 patients, for an overall postoperative hardware infection rate of $3.6 \%$. This rate dropped to $2.6 \%$ when we excluded early procedures in which the bur hole had been drilled outside of the MRI room followed by transport into the magnet room.

\section{Rationale for Performing DBS Implantation Outside of an Operating Room}

Interventional MRI-guided DBS electrode implantation is a promising technique. It has demonstrated high targeting accuracy ${ }^{16}$ and clinical outcomes comparable to those for conventional implantation approaches..$^{15,16}$ The method allows patients to avoid an awake surgical procedure, making the therapy available to a wider range of cases including pediatric patients. The number of MRI scanners in the United States was 3265 in 1995 and 7810 in $2007,,^{12}$ indicating wide system availability for these types of procedures. However, only a very small fraction of these systems exist in an operating room environment, and many of these are not ideal for high-resolution basal ganglia imaging. Thus, the requirement that an MRI suite meet operating room standards would place a significant barrier to the adoption of the iMRI-guided technique.

\section{Infection Risk in iMRI Technique Versus Standard DBS Techniques}

The incidence of infection we found for iMRI-guided procedures is comparable to that reported for DBS surgeries performed in traditional operating rooms. ${ }^{1,5,14,17}$ Prior studies have used variable postoperative windows during which an infection can be attributed to DBS implantation. We used a postoperative window of 6 months to include more slowly evolving infections. The National Surgical Quality Improvement Program (NSQIP) standard requires an infection to appear within 30 days to qualify as a surgical infection. Tolleson et al. reported a substantially lower infection rate (2.0\%) when applying the NSQIP standard, as compared with that found if a longer window was used (5.8\%). ${ }^{17}$ Sillay et al. established a 6-month window for postoperative hardware infection-because hardware infections occurring beyond 6 months were associated with delayed device erosion following a period of complete healing ${ }^{14}$-and found a hardware infection rate of $4.5 \%$. The Veterans Affairs cooperative study of 6-month outcomes in 121 patients who had received bilateral DBS electrodes reported an infection rate of $9.9 \% .^{18}$ Thus, the 6-month infection rates in the present study are well within the reported range for DBS implantation procedures performed in conventional operating room environments. This remains true even if we consider upper bounds for our infection rates, at the $95 \%$ confidence level, based on probability theory.

\section{Factors Influencing the Risk of Infection}

Effective execution of sterile practice plays a critical role in reducing infection risk. This assertion was borne out in our early studies, in which our workflow involved a complex 2-stage draping procedure, with an intervening transfer of the patient to the adjacent MRI room, and resulted in 2 infections in our first 10 cases.${ }^{16}$ These infections were associated with the frontal incision site. Since adopting our current 1-stage draping procedure entirely within the MR bore, all infections have been consistent with a point of origin near the IPG. Prior studies have also revealed that the IPG is the most common site for infection, followed by the parietal lead extender connection, then the frontal incision. ${ }^{14}$ With the iMRI technique, the extender wire and IPG are implanted in a second surgical procedure 1-3 weeks after electrode implantation, a separate and distinct source for potential infection. Thus, it appears that the utilization of a diagnostic MRI suite, in combination with prudent sterile practices applied by experienced personnel, does not increase the infection incidence in patients undergoing DBS electrode implantation.

A potential reason for the relatively low incidence of infection during these procedures may relate to surgical duration. Prior studies of infection incidence have correlated longer operating times with higher infection rates during tumor resection ${ }^{9}$ and CSF shunt placement. ${ }^{8}$ Interventional MRI-guided DBS implantations are time efficient as compared with conventional DBS implantation with physiological monitoring. Our NexFrame cases (2010 and earlier) had skin incision to skin closure times that averaged $224 \pm 28$ minutes for bilateral procedures and $211 \pm 59$ minutes for unilateral procedures. Our ClearPoint cases (2010 and later) improved on these times and have averaged $184 \pm 28$ minutes for bilateral procedures and $137 \pm$ 25 minutes for unilateral procedures. These times are substantially shorter than those reported for physiologically guided DBS implantation approaches. However, the small sample size prevents any meaningful statistical analysis correlating risk factors. Indeed, a Norwegian study that analyzed 588 surgical DBS procedures and reported 33 infections still failed to identify any significant risk factors. ${ }^{3}$ Thus, whether the shorter duration of iMRI-guided DBS implantation contributes to a reduction in the risk of infection remains conjecture.

An operating room nursing team (scrub nurse and circulating nurse) assisted with all of our procedures. It is possible that the infection rate would have been higher if procedures had been performed with nursing assistants who did not routinely work in an operating room environment. Finally, in 1 recent patient there was evidence of MSSA on a nasal swab taken several months prior to surgery. The same bacterium was ultimately present in the postoperative infection. At our center we screen patients for methicillin-resistant $S$. aureus on nasal swabs as part the preoperative workup, and if the swab is positive, decolonization treatment is prescribed. Recently we have begun screening for MSSA as well. Justification for decolonizing prior to surgery is emerging, ${ }^{4}$ which may help to reduce the incidence of infection in patients with bacterial colonization.

\section{Conclusions}

Infection risk when implanting DBS electrodes in a di- 
agnostic MRI suite is comparable to that reported for large series of DBS electrodes implanted in a regular operating room environment. The use of standard sterile techniques similar to those used in a regular operating room, practiced by surgical and nursing teams highly experienced in device implantation, may be important in achieving an acceptable infection risk.

\section{Acknowledgments}

We thank Jeff Myers, ST, and Nicholas Jeung, AART(MR), for their efforts in establishing safe and effective sterile techniques in a diagnostic MRI suite.

\section{References}

1. Baizabal Carvallo JF, Mostile G, Almaguer M, Davidson A, Simpson R, Jankovic J: Deep brain stimulation hardware complications in patients with movement disorders: risk factors and clinical correlations. Stereotact Funct Neurosurg 90:300-306, 2012

2. Benazzouz A, Breit S, Koudsie A, Pollak P, Krack P, Benabid AL: Intraoperative microrecordings of the subthalamic nucleus in Parkinson's disease. Mov Disord 17 (Suppl 3):S145S149, 2002

3. Bjerknes S, Skogseid IM, Sæhle T, Dietrichs E, Toft M: Surgical site infections after deep brain stimulation surgery: frequency, characteristics and management in a 10 -year period. PLoS One 9:e105288, 2014

4. Bode LG, Kluytmans JA, Wertheim HF, Bogaers D, Vandenbroucke-Grauls CM, Roosendaal R, et al: Preventing surgical-site infections in nasal carriers of Staphylococcus aureus. N Engl J Med 362:9-17, 2010

5. Fenoy AJ, Simpson RK Jr: Risks of common complications in deep brain stimulation surgery: management and avoidance. J Neurosurg 120:132-139, 2014

6. Hall WA, Truwit CL: Intraoperative MR-guided neurosurgery. J Magn Reson Imaging 27:368-375, 2008

7. ISO: Cleanrooms and associated controlled environmentsPart 1: Classification of air cleanliness. ISO 14633-1:2015. Geneva: International Organization for Standardization, 1999

8. Korinek AM, Fulla-Oller L, Boch AL, Golmard JL, Hadiji B, Puybasset L: Morbidity of ventricular cerebrospinal fluid shunt surgery in adults: an 8-year study. Neurosurgery 68:985-995, 2011

9. Korinek AM, Golmard JL, Elcheick A, Bismuth R, van Effenterre R, Coriat $\mathrm{P}$, et al: Risk factors for neurosurgical site infections after craniotomy: a critical reappraisal of antibiotic prophylaxis on 4,578 patients. Br J Neurosurg 19:155-162, 2005

10. Larson PS, Starr PA, Bates G, Tansey L, Richardson RM, Martin AJ: An optimized system for interventional magnetic resonance imaging-guided stereotactic surgery: preliminary evaluation of targeting accuracy. Neurosurgery 70 (1 Suppl Operative):95-103, 2012
11. Martin AJ, Larson PS, Ostrem JL, Keith Sootsman W, Talke P, Weber OM, et al: Placement of deep brain stimulator electrodes using real-time high-field interventional magnetic resonance imaging. Magn Reson Med 54:1107-1114, 2005

12. National Center for Health Statistics: Health, United States, 2011: With Special Feature on Socioeconomic Status and Health. Hyattsville, MD: US Department of Health and Human Services, 2012

13. Ninomura P, Bartley J: New ventilation guidelines for healthcare facilities. ASHRAE J 43:29-33, 2001

14. Sillay KA, Larson PS, Starr PA: Deep brain stimulator hardware-related infections: incidence and management in a large series. Neurosurgery 62:360-367, 2008

15. Starr PA, Markun LC, Larson PS, Volz MM, Martin AJ, Ostrem JL: Interventional MRI-guided deep brain stimulation in pediatric dystonia: first experience with the ClearPoint system. J Neurosurg Pediatr 14:400-408, 2014

16. Starr PA, Martin AJ, Ostrem JL, Talke P, Levesque N, Larson PS: Subthalamic nucleus deep brain stimulator placement using high-field interventional magnetic resonance imaging and a skull-mounted aiming device: technique and application accuracy. J Neurosurg 112:479-490, 2010

17. Tolleson C, Stroh J, Ehrenfeld J, Neimat J, Konrad P, Phibbs F: The factors involved in deep brain stimulation infection: a large case series. Stereotact Funct Neurosurg 92:227-233, 2014

18. Weaver FM, Follett K, Stern M, Hur K, Harris C, Marks WJ $\mathrm{Jr}$, et al: Bilateral deep brain stimulation vs best medical therapy for patients with advanced Parkinson disease: a randomized controlled trial. JAMA 301:63-73, 2009

\section{Disclosures}

The authors received grant funding from the following sources: NIH R21 EB008888, UC Discovery Grant No. 156321, MRI Interventions (research contract, A.J.M., J.L.O., P.S.L., and P.A.S.), Medtronic (research contract, P.S.L. and P.A.S.), and Image Guided Neurologics (research contract). Dr. Starr is a consultant for Boston Scientific.

\section{Author Contributions}

Conception and design: Martin, Larson, Ostrem, Starr. Acquisition of data: Martin, Larson, Ziman, Levesque, Starr. Analysis and interpretation of data: Martin, Larson, Ostrem, Starr. Drafting the article: Martin, Starr. Critically revising the article: Martin, Larson, Ziman, Levesque, Ostrem, Starr. Reviewed submitted version of manuscript: all authors. Approved the final version of the manuscript on behalf of all authors: Martin. Administrative/ technical/material support: Volz. Study supervision: Martin, Starr.

\section{Correspondence}

Alastair J. Martin, Department of Radiology, University of California, San Francisco, 505 Parnassus Ave., San Francisco, CA 94143. email: alastair.martin@ucsf.edu. 